\title{
Dictynna
}

Dictynna

Revue de poétique latine

1 | 2004

Varia

\section{Tradition, vraisemblance et autorité fictionnelle}

\section{Alain Deremetz}

\section{(2) OpenEdition}

Journals

Édition électronique

URL : http://journals.openedition.org/dictynna/159

DOI : 10.4000/dictynna.159

ISSN : 1765-3142

Édition imprimée

ISBN : 2-84467-088-1

Référence électronique

Alain Deremetz, «Tradition, vraisemblance et autorité fictionnelle », Dictynna [En ligne], 1 | 2004, mis en ligne le 15 novembre 2010, consulté le 10 septembre 2020. URL : http://journals.openedition.org/ dictynna/159; DOI : https://doi.org/10.4000/dictynna.159

Ce document a été généré automatiquement le 10 septembre 2020.

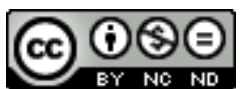

Les contenus des la revue Dictynna sont mis à disposition selon les termes de la Licence Creative Commons Attribution - Pas d'Utilisation Commerciale - Pas de Modification 4.0 International. 


\title{
Tradition, vraisemblance et autorité fictionnelle
}

\author{
Alain Deremetz
}

\section{Je auctorial et vraisemblance pragmatique}

1 Exploitant une remarque de Marthe Robert ${ }^{1}$ qui opposait le roman colonisateur du XIX siècle, où le narrateur hétérodiégétique se voit attribuer « des capacités de perception illimitées ", et le roman "classique » où il «faut feindre de ne rien imaginer » et se référer "à une quelconque autorité extérieure ", C. Cavillac ${ }^{2}$ observe que le pacte romanesque ${ }^{3}$ sur lequel ce dernier repose «doit inclure, pour être recevable, un protocole pragmatique » chargé de « justifier la performance narrative». Elle identifie ainsi, à côté de la vraisemblance empirique des énoncés (« qui porte sur la conformité à l'expérience commune, mesurée à l'aune de la raison et/ou de l'opinion») et de la vraisemblance diégétique (qui porte "sur la cohérence de la mise en intrigue »), la vraisemblance pragmatique " qui concerne la fictivité de l'acte de narration », i.e. tout ce qui, dans un tel récit, concerne «le mode d'information du narrateur et les circonstances de l'énonciation »; et elle ajoute que cette vraisemblance pragmatique «constitue la pièce maîtresse et la condition nécessaire (mais non suffisante) de l'autorité fictionnelle, avant tout comprise comme autorité de la voix narrative ». Pour être reçue, toute fiction devait feindre de ne pas être fictive et de ne rien rapporter qui «n'ait été vécu par l'auteur, su par la vox populi (i.e. la fama) ou connu par des documents ${ }^{4} »$.

2 Si liée qu'elle paraisse au développement du roman classique, cette exigence de vraisemblance pragmatique me semble avoir pourtant une longue histoire, aussi longue sans doute que celle de la littérature. Après un premier chapitre que j'ai consacré à cette notion dans le genre romanesque ${ }^{5}$, il ne me paraît pas inutile de tenter d'en écrire un second en élargissant le point de vue à certains genres fictionnels antiques, notamment l'élégie et la satire, mais également, aussi paradoxal que cela puisse paraître, à des genres « factuels » tels que l'histoire ou l'épopée romaines. 


\section{Vraisemblance pragmatique et épopée : le paradoxe}

3 En disant qu'il est paradoxal de parler de vraisemblance pragmatique à propos de l'histoire et de l'épopée (et bien sûr tout particulièrement de l'épopée historique romaine), je ne fais rien d'autre que reprendre provisoirement à mon compte la position prise par C. Cavillac quand elle affirme que cette exigence ne concerne que les récits où « la vraisemblance mimétique s'étend à la sphère du privé, du personnel, de l'humble, de l'obscur ", le narrateur de tels récits privés étant toujours tenu de préciser ce qui a rendu son récit possible, alors même qu'il ne fait que l'inventer. Pour ces deux genres, au contraire, la question ne se poserait pas «puisque, dit-elle, l'un porte sur le vrai (du moins en droit) et que l'un comme l'autre sont voué(e) s à retracer des actions attestées et mémorables » (p. 26). Ainsi, à la différence de l'auteur de roman, l'auteur d'une Histoire " peut sans choquer outre mesure ${ }^{6}$ faire œuvre de poète en interpolant dans le récit d'une vie illustre discours ou harangues de son cru, parce qu'il s'agit là d'actes publics» (p. 27). C'est du reste bien ce qu'atteste Quintilien qui pense que l'orateur (ou l'historien) peut inventer des faits à condition qu'ils «aient un air de vérité qui ne jure ni avec la personne, ni avec le lieu, ni avec le temps, et que tout soit vraisemblable dans l'accomplissement comme dans l'ordre des faits ${ }^{7}{ }^{\prime}$.

4 Quant au poète épique, précise-t-elle, "s'il s'accorde des pouvoirs beaucoup plus étendus que l'historien, contant des faits qui n'ont pas eu de témoins ou dévoilant les passions et les querelles des dieux ", c'est parce qu'il peut se réclamer de la Muse qui " dit les causes » : « toute l'action épique se déroule au regard des divinités, ce qui suffit à en autoriser la narration par "un âme possédée ", soumise à l'autorité d'une voix extérieure, omnisciente, comme l'évoquent la scène célèbre du début de la Théogonie d'Hésiode ou, plus largement, le topos de la possession divine (en relation avec Apollon et/ou Dionysos). Et elle cite à l'appui de son analyse le célèbre passage du Satiricon où Pétrone fait dire au Eumolpe :

Ce ne sont pas les événements eux-mêmes qui doivent être exprimés dans les vers les historiens le font beaucoup mieux -, mais c'est, à travers les épisodes, les interventions divines, les développements empruntés à l'arsenal de la fable, une inspiration autonome qui doit se donner carrière, de telle sorte que l'on ait l'impression d'une révélation issue d'une âme possédée plutôt que d'un récit authentique et garanti par des témoignages fidèles ${ }^{8}$.

5 À la narration more historico, Pétrone oppose la narration more epico, ces deux narrations ne se distinguant pas sur le plan du référent - il s'agit dans les deux cas du récit d'actions attestées ou attestables -, mais bien sur celui du style et des procédés de la véridiction (i.e. du faire croire) qui ressortissent à deux postures narratives différentes, l'une reposant sur la garantie de l'inspiration et l'autre sur celle du témoignage, voire du document. Il n'en reste pas moins vrai que, pour être crus, l'historien et le poète épique font également dépendre leur parole d'un locuteur premier, censé avoir eu accès à la vérité, en raison soit de sa nature, soit de sa proximité avec les événements rapportés. 


\section{Vraisemblance et genres fictionnels}

6 Je voudrais maintenant évoquer rapidement quelques œuvres antiques qui semblent donner raison à $\mathrm{C}$. Cavillac quand elle affirme que c'est dans les récits fictionnels entrant dans la sphère du privé et de l'humble que se manifeste l'exigence de vraisemblance pragmatique. Celui qui, parmi les genres, en offre la meilleure illustration est le roman qui a recours à au moins deux types de protocole pragmatique. Le premier, que je trouve dans les premiers mots du roman de Chariton, Chéréas et Callirhoé, est emprunté à l'historiographie ${ }^{9}$ :

Moi, Chariton d'Aphrodise, secrétaire du rhéteur Athénagore, je vais raconter une

histoire d'amour (pathos erotikon) qui est arrivée à Syracuse.

7 L'identité de l'auteur et/ou du narrateur ne se trouve que dans la première phrase, qui est une sorte de proimion contenant une sphragis, comparable à celle d'Hésiode dans la Théogonie ou à celle d'Hérodote dans ses historiai, en même temps que l'ouverture du récit fictif. Le pacte narratif ainsi constitué peut se lire comme un acte de langage qui est une déclaration constituant une invitation à écouter un récit dont les événements sont par là même supposés avoir existé, car déclarer revient à créer un état de fait. $\mathrm{Ce}$ faisant, le narrateur se donne un univers de référence, le monde possible où se déroulera la fiction. Mais une fois posé ce monde fictionnel, il doit le rendre vraisemblable c'est-à-dire ici le déguiser sous l'apparence du discours référentiel factuel, historique.

Ce déguisement, en effet, est manifeste : le romancier parle à la première personne, se posant comme sujet de son discours à la manière de Thucydide ou d'Hérodote :

L'Athénien Thucydide a consigné par écrit comment s'est déroulée la guerre qui opposa les Péloponnésiens et les Athéniens.

écrit Thucydide et Hérodote ${ }^{10}$ avait usé d'un procédé semblable :

Voici l'exposé de l'enquête (apodexis historiès) effectuée par Hérodote de Thourioi,

afin que les événements humains ne disparaissent pas avec le temps.

Comme Thucydide et Hérodote, Chariton inscrit en tête de son ouvrage son nom, son pays d'origine (il y a donc présomption de réalité) et son statut de scripteur. Il se présente comme quelqu'un sachant écrire (il est hupographeus, un secrétaire qui écrit sous la dictée d'un autre) rapportant un événement passé. Chariton emploie d'ailleurs le verbe diegesomai qui rappelle les discours des orateurs annoncés comme devant se faire oralement devant l'auditoire, discours qui peuvent raconter des événements qui se sont déroulés ou qui semblent l'avoir fait. L'expression pathos erotikon, quant à elle, ne permet pas de lever l'ambiguïté, cette passion ayant pu ou non être réelle. Du reste, l'emploi chez Chariton de genomenon, qui rappelle le ta genomena d'Hérodote, qualifie le récit comme authentique et rapportant des faits réels ${ }^{11}$.

Le second type, celui-là même que dénonce et refuse d'utiliser Lucien dans l'Histoire Véritable, apparait dans les Métamorphoses d'Apulée, et sera repris dans les romans picaresques espagnols (Lazarillo de Tormes et Guzman de Alfarache). Il repose sur l'artifice autobiographique, le narrateur racontant qu'après avoir vécu sous la peau d'un âne (ce qui justement lui a permis d'accéder incognito à toutes les situations et de recueillir toutes sortes de récits possibles), il vit la vie édifiante d'un pêcheur repenti (comme Guzman) que sa vie de tribulations a rendu « sinon plus sage, du moins riche de savoir, $9,13)$ et qui, une fois initié aux mystères d'Isis et d'Osiris, se rend à Rome où il apprend la langue latine puis plaide au forum ; et c'est là qu'il raconte en latin son aventure, qui 
se trouve être une œuvre littéraire dont le genre est célèbre et qui connait un précédent. Dans ce roman, comme dans celui de Pétrone, «les aventures d'anti-héros comme Lucius-l'âne et Encolpe le marginal requièrent le témoignage personnel, parce que nul autre qu'eux-mêmes ne saurait rendre compte d'une vie qui n'est pas inscrite dans l'Histoire. En pareil cas, le problème de l'information du narrateur est, pour l'essentiel, résolu par l'artifice autobiographique...» (Cavillac, p. 28). Est donc importante la question des «circonstances de l'énonciation" qui, dans les Métamorphoses, est résolue précisément sur le plan narratif par la métamorphose du narrateur grec en scripteur latin, ceci après une initiation et un voyage, comparables à ceux d'Énée, qui assurent le transfert du genre milésien en terre latine.

Dans ce récit, comme dans les romans picaresques qui en dérivent, la "vraisemblance pragmatique semble éliminer toute trace d'une autre instance d'énonciation ${ }^{12}$ » que Lucius, le héros et le narrateur de son aventure, qui est assez cultivé et maître de la langue pour produire un tel récit typique de l'éloquence de la seconde sophistique. Le «je narré» finit par rejoindre le «je narrant» et s'identifier à lui, presque parfaitement; l'œuvre est ainsi sans auteur (ou presque si l'on admet le lapsus de 11, 27), même si celui-ci est censé avoir mis son nom sur les volumina contenus dans la boîte.

Ce protocole pragmatique qu'assure le recours à l'artifice autobiographique ${ }^{13}$ est aussi commun dans l'élégie que dans le roman. Prétendant, en effet, relater une histoire privée qui se déroule hors du champ de l'Histoire, le narrateur-amant lui aussi n'a pas d'autre moyen de justifier son information que le témoignage personnel. C'est ce qui explique pourquoi Tibulle, Properce et Ovide se sont employés à ancrer leurs poèmes dans la réalité vécue, en s'inventant une histoire personnelle remplie d'expériences vraisemblables : la rencontre, l'innamoramento, l'entreprise de séduction, tout cela en des lieux et en des circonstances reconnaissables.

13 C'est d'ailleurs ce même procédé qu'Ovide dénude, comme à son habitude, au début de son Art d'Aimer où il prétend que son traité ne procède pas, comme celui d'Hésiode, d'une inspiration divine :

Je ne mentirai pas, Phébus, en prétendant que tu m'as inspiré cet art; ce n'est pas non plus le chant des oiseaux du ciel qui m'a instruit et je n'ai pas rencontré Clio et ses sœurs pendant que je gardais les troupeaux dans tes vallées, Ascra...;

mais qu'il est le fruit de l'expérience acquise par un homme qui a subi l'amour et qui a été la victime ou le bénéficiaire de tous les événements qu'il rapporte :

C'est l'expérience (usus) qui anime cet ouvrage: obéissez au prophète qui s'y connaît (perito). Je chanterai la vérité ; favorise mon entreprise, mère d'Amour. (1, 25-29).

14 À la proclamation solennelle, commune dans la grande poésie, que seul est véridique le chant inspiré par les dieux, Ovide substitue donc ici une autre garantie de véridicité, celle de la compétence (peritia) que donne l'expérience (usus) ${ }^{14}$. Ce faisant, il conclut avec son lecteur un nouveau "pacte d'illusion consentie ", plus vraisemblable parce qu'il convient mieux à son sujet, l'art de la conquête amoureuse, et au genre choisi, l'élégie ; et ce nouveau pacte exige lui-même un protocole pragmatique qui rompt avec l'usage de la grande poésie, protocole qui fait reposer son autorité fictionnelle sur l'expérience que lui aurait fournie sa propre vie. Alors même, comme le précise C. Cavillac, qu'il ne «s'agit pour lui que de sacrifier de façon plus ou moins ludique à une formalité », le narrateur élégiaque s'estime tenu de " justifier sa performance narrative au nom du principe qu'on ne peut rapporter que des choses qu'on a apprises » ou 
vécues. Il feint ainsi de faire croire que tout ce qu'il raconte est vrai, et fait comme si ce qu'il dit avoir appris par expérience n'existait pas dans la tradition poétique à laquelle il puise pourtant allégrement. En d'autres termes, le pacte élégiaque qu'il instaure repose tout entier sur un contrat de véridiction paradoxal qui inclut un protocole pragmatique dont la modalité est assimilable à celle d'une « fiction du non-fictif ${ }^{15}$ ». Les lecteurs antiques se sont-ils laissés abuser, comme nombre de commentateurs modernes, par un tel stratagème énonciatif? Je ne le crois pas, même si certaines thèses sur l'exil d'Ovide laissent supposer le contraire. Pour ma part, je penserais plutôt qu'Ovide fut pris à son propre piège, comme l'arroseur arrosé.

Tel me paraît être aussi le cas de la satire qui, plus encore que l'élégie, est liée par un contrat de "pseudo-véridiction ", les poètes donnant au lecteur l'assurance qu'ils ne font que décrire la réalité quotidienne et relater des événements dont ils ont été les témoins ou les victimes.

Participe de cette mise en scène l'autoportrait qu'Horace dresse de lui-même dans la satire $1,6^{16}$ où il évoque sa première rencontre et sa première conversation avec Mécène, conversation au demeurant fort brève comme doit l'être tout sermo :

Un jour l'excellent Virgile, après lui Varius t'ont dit ce que j'étais. Quand je fus venu devant toi, alors, prononçant quelques mots entrecoupés, car la timidité, qui arrête la langue, m'empêchait d'en dire davantage, je ne me vante point d'être né d'un père illustre, ni de faire le tour de mes domaines porté sur un cheval de Saturium, mais j'explique ce que j'étais. Tu me réponds, selon ton habitude, en quelques mots; je m'en vais; tu me rappelles neuf mois plus tard, et tu m'invites à compter au nombre de tes amis. C'est beaucoup, à mes yeux, de t'avoir plu, à toi qui, pour distinguer l'honnête homme de l'homme sans honneur ne regardes point l'illustration du père, mais la netteté de la vie et du cœur.

Et, pourtant, si ma nature, droite d'ailleurs, n'est entachée que de défauts médiocrement graves et en petit nombre, comparables à des verrues qu'on trouverait éparses sur un beau corps, si personne ne peut sans mentir me reprocher ni avarice, ni tenue sordide, ni basse débauche, si, pour faire mon propre éloge, ma vie est nette et sans reproche, si je suis cher à mes amis, je le dois à mon père, qui, pauvre d'un maigre petit bien, ne voulut pas m'envoyer à l'école de Flavius...

Cet autoportrait moral qui est placé sous la caution de Mécène (dont le laconisme est bien dans le ton de la satire) sert à qualifier Horace en locuteur autorisé du genre et à asseoir son autorité fictionnelle sur la garantie de la simplicité, de l'honnêteté et de la sincérité (qualités qui sont nécessaires à l'établissement du pacte d'illusion consentie).

Mais, pour garantir que ses satires sont bien, comme les comédies, des «miroirs de la vie », le poète ne se limite pas à assurer qu'ils procèdent d'une autopsie authentique : il leur attribue aussi d'autres traits stylistiques, qui sont ceux du sermo pedester, c'est-àdire pour reprendre ce qu'a fort justement écrit à ce propos $\mathrm{M}$. Citroni ${ }^{17}$, la nonpoéticité, la dimension non artistique et le caractère acanonique d'un style qui rase le sol. Une telle posture implique un rapport ambigu avec la tradition. Alors que la satire, en tant qu'elle s'inscrit dans un genre établi, exige de la part du poète qu'il respecte (ou dénonce) des règles héritées et puise à une topique convenue, en tant qu'elle prétend être un discours efficace sur le monde contemporain, doit masquer son rapport à la tradition et se présenter, par exemple, comme le fruit de la colère ou de l'indignation qui naît spontanément devant un spectacle indigne, cela afin de provoquer un sentiment analogue chez le lecteur. J'ai dit « masquer » et non " occulter » bien sûr, car il est nécessaire à la stratégie du poète que le protocole énonciatif qu'il emploie puisse être reconnu comme tel. 
19 L'exemple par lequel je voudrai illustrer brièvement mon propos est celui que m'a inspiré l'excellente analyse que, dans une thèse récemment soutenue, J. Soler a consacrée à la satire $\mathrm{V}$, dite du voyage à Brindes. Tout dans ce récit semble être le témoignage précis d'un voyage réel : les circonstances en sont attestées historiquement (vv. 28 et 29, et cela malgré le caractère allusif de la formule « habitués à réconcilier des amis divisés »), les membres sont tous des personnages en vue de la cour impériale, l'itinéraire est détaillé avec ses étapes et ses anecdotes, bref tout y donné comme la transcription de notes prises chemin faisant. Pourtant de nombreux indices invitent le lecteur à lire dans cette relation la reprise parodique d'un récit-modèle, le célèbre Iter Siciliense de Lucilius, père fondateur du genre; de sorte que ce qu'Horace exprime dans ce récit, c'est le mode de composition de la satire et le style du genre qui est à l'image $\mathrm{du}$ cheminement laborieux d'anti-héros, paresseux, ophtalmiques et dyspepsiques montés sur le dos d'un âne allant au pas et toujours prêts à faire halte dans une bonne auberge. Dans cet art poétique en acte, je devrais dire, chemin faisant, où il délivre sa conception du genre, Horace s'amuse à jouer avec les contraintes pragmatiques que lui impose le genre satirique, celles d'assumer une situation singulière et d'endosser le rôle vraisemblable du voyageur ; et peu importe que le voyage ait été réel. Et l'on pourrait dire la même chose de nombre de satires, celles du fâcheux ou de la consultation chez Trébatius, par exemple.

\section{La vraisemblance chez les théoriciens} consentie ", la référence à la tradition joue un tout autre rôle dans l'épopée. C'est ce que je vais examiner maintenant après avoir essayé de répondre à une question: l'épopée relevait-elle pour les anciens du factuel ou du fictionnel ? Sur ce problème complexe, voire insaisissable, je ne dirai que quelques mots.

I faut tout d'abord procéder à une mise au point : la notion de fiction, au sens où nous l'entendons aujourd'hui, existait-elle chez les Anciens? Comme l'a rappelé J. Boulogne ${ }^{18}$, pour Aristote, « le rôle du poète est de dire non pas ce qui a lieu réellement (c'est le rôle du chroniqueur), mais ce pourrait avoir lieu dans l'ordre du vraisemblable et du nécessaire » (Poétique, 9, 51a). Le vraisemblable peut donc être vrai ou faux, attestable ou non, mais ce qui importe le plus au poète est moins l'authenticité de ce qu'il rapporte que la manière d'en composer le récit ${ }^{19}$; ce qui revient à dire que la vraisemblance diégétique compte plus pour lui que la vraisemblance empirique: une œuvre poétique, qu'elle soit fictionnelle ou factuelle, est toujours une composition. Du reste, il admettait que la frontière entre les narrations épique et historique soit transgressée par le poète qui «peut composer un poème sur des événements réellement arrivés, car rien n'empêche que certains événements réels ne soient de ceux qui pourraient arriver dans l'ordre du vraisemblable et du nécessaire ${ }^{20} \%$. Je serais tenté d'interpréter ces mots de la manière suivante : un récit, que le sujet de l'histoire qu'il raconte soit ou non authentique, est une fiction, dès lors qu'il respecte les règles de l'art poétique.

La tradition péripatéticienne ultérieure va préciser ce statut de la composition poétique en proposant une classification des récits qui recourt, de manière semble-t-il plus claire, au critère de vraisemblance empirique et référentielle. C'est la tripartition que l'on trouve chez Asclépiade de Myrlaea ${ }^{21}$ qui oppose l'historia (l'enquête), qui est du côté 
de la vérité attestée, le plasma, qui est une fiction relevant du vraisemblable, et le mythos qui est une fiction mensongère relevant de l'invraisemblable. Dans laquelle de ces trois catégories rangeait-il l'épopée ? il est difficile de le dire, ne serait-ce que parce qu'aucun genre ne leur est associé. Sans doute faut-il penser que cette classification porte sur les sujets et non sur les genres, et que les œuvres d'un genre tel que l'épopée peuvent appartenir aux trois catégories.

Les théoriciens latins reprendront cette tripartition en lui faisant subir deux infléchissements. Le premier est qu'ils semblent accorder une place plus grande encore au critère de vérité, en raison peut-être, comme le croit C. Cavillac ${ }^{22}$, du glissement conceptuel qu'induit « le passage du terme eikos au latin veri similis: de ce qui est eikos, " plausible ", " attendu ", on passe à ce qui est veri similis, "semblable au vrai ». Il faut donc que le vrai soit connaissable pour qu'il y ait du vrai-semblable, du semejante a verdad; mais s'il y a du vrai-semblable, comment le vrai est-il (re)connaissable?». Le second infléchissement est qu'une mise en rapport partielle apparaît entre les genres et les trois catégories, comme on peut le lire au livre II de l'Institution oratoire:

La narration, dit-il..., exception faite du type judiciaire, dit-il, revêt, nous l'avons appris, trois formes : la 'fable' (fabula), que l'on rencontre dans les tragédies et les poèmes, qui est éloignée de la vérité et même d'une apparence de vérité; le 'scénario' (argumentum) de la comédie, qui est une invention fausse, mais vraisemblable; l'histoire' (historia) qui contient le récit (expositio) d'une action réelle ; quant à la narration poétique, nous l'avons réservée aux grammairiens : par suite, le rhéteur doit traiter d'abord de narrations historiques, qui ont d'autant plus de forces qu'elles sont plus vraies ${ }^{23}$.

Chez lui la tripartition cache donc une bipartition : aux narrations fausses qui relèvent de la poésie s'opposent les vraies, l'historique et la judiciaire, la première préparant à la seconde. Comme je l'ai exposé ailleurs, la frontière qu'il établit, selon leur rapport au réel, entre ces deux grands types de narrations, que, pour reprendre la terminologie de Genette $^{24}$, je qualifierai de fictionnelles et de factuelles, coïncide pour lui exactement avec celle qu'il veut restaurer entre les devoirs respectifs du grammairien et du rhéteur. Or, la ligne de partage ainsi tracée entre les professions est également disciplinaire, puisqu'elle est précisément celle qui sépare les définitions que, depuis Aristote, la poétique et la rhétorique donnent respectivement de la narration ${ }^{25}$.

Il reste, comme je l'ai déjà dit, que, pour Quintilien et pour Aristote, cette frontière peut être transgressée, aussi bien par le poète qui "peut composer un poème sur des événements réellement arrivés ${ }^{26} . . . "$, que par l'orateur (ou l'historien), qui peut inventer des faits à condition qu'ils "aient un air de vérité qui ne jure ni avec la personne, ni avec le lieu, ni avec le temps, et que tout soit vraisemblable dans l'accomplissement comme dans l'ordre des faits ${ }^{27} »$. En conséquence, bien que la réalité des faits rapportés soit le critère affiché de la différenciation des deux modes de narration, une même objectif de vraisemblance est exigé du poète et de l'orateur (ou de l'historien) quand ils racontent.

Il faut donc admettre que, pour lui, l'épopée se répartit entre les deux catégories extrêmes, selon que le sujet appartient, comme c'est le cas à Rome, au mythe ou à l'histoire ; et sans doute est-ce aussi l'opinion de Cicéron telle qu'on peut la déduire, me semble-t-il, du passage un peu énigmatique du De inventione (I, 27) où l'orateur avait déjà repris la tripartition canonique, mais en l'appliquant explicitement au seul champ poétique. C'est ainsi que la Médée, une tragédie de Pacuvius, illustrait pour lui la fabula et l'Andrienne, comédie de Térence, l'argumentum; et pour illustrer l'historia, il choisit 
les Annales d'Ennius ${ }^{28}$, si l'on accepte l'attribution à cet auteur du vers Appius indixit Carthaginiensibus bellum. L'épopée, qui est substituée au genre historiographique, relève donc clairement de la vérité historique ; mais il s'agit de l'épopée historique romaine.

$\mathrm{Au}$ terme de cette rapide enquête, il ressort que :

- l'épopée peut se trouver du côté du factuel ou du fictionnel, selon l'époque (cf. l'épopée mythographique), mais l'épopée romaine est du côté du factuel ;

- ce qui distingue l'histoire de l'épopée se pose moins en termes de vraisemblance empirique que diégétique, c'est-à-dire en termes de posture narrative ;

- le poète épique, comme l'historien, peuvent également introduire des éléments fictifs dans des œuvres dont le référent est relève du factuel et donc mettre en œuvre une poétique ;

- leur stratégie commune sera toujours de rendre croyables les faits qu'ils racontent, qu'ils soient authentiques ou fictifs.

\section{Épopée et vraisemblance}

négligeaient-ils ? - où le genre épique s'est largement nourri de sujets historiques, mais la principale tient sans doute au fait que l'épopée latine, tout en continuant à revendiquer son appartenance à l'oralité et à la mémoire des Muses, s'inscrit à la suite de l'épopée grecque - notamment alexandrine - (à laquelle elle puise ses formes et parfois ses sujets) dans l'espace de la scripturalité. C'est ainsi que le simulacre de la muse $^{30}$, par lequel le poète dissimule la manipulation sociale dont il est l'objet (il travaille sur commande), finit par ne plus devenir qu'une marque générique de la haute poésie, un élément obligé de son code. Le vates devient désormais poeta doctus, conscient du fait que son dire dépend de l'existence d'une tradition littéraire complexe, enregistrée dans des textes consultables, avec laquelle il doit composer, dont il prétend arrêter le déploiement et qu'il prétend régler pour l'avenir, en l'unifiant dans un tout organique.

31 Le narrateur se voit contraint d'établir l'autorité de sa voix narrative (ou du moins en expliciter le statut) et d'assurer la vraisemblance pragmatique de son récit (comment un tel récit est-il possible ?) en respectant à cette fin un protocole dont les modalités sont variables selon les genres. Parmi les principaux éléments constitutifs de ce protocole pragmatique, je me limiterai à examiner ceux qui, s'ils existent, renvoient à ce que le poète dit, explicitement ou de manière reconnaissable, des sources sur 
lesquels il fonde la vraisemblance voire la vérité de son récit. Pour lui, «assurer la vraisemblance pragmatique de sa narration" revient à expliciter le lien qu'elle entretient avec la tradition dont elle hérite, c'est-à-dire à désigner d'une manière ou d'une autre les auteurs et les œuvres qui selon lui en font partie; et c'est ce qu'il fait d'ordinaire en recourant à divers procédés, explicites et implicites (allusifs), de citations, d'allusions, de reprises ou de reformulations d'œuvres antérieures qui contribuent à le qualifier comme locuteur autorisé et compétent, parce qu'informé31.

32 J'ai conscience, en abordant cette question, d'ouvrir de manière imprudente et tardive le lourd et complexe dossier qui a été consacré ces dernières années à l'intertextualité. Ce dossier se résume, me semble-t-il, à deux questions essentielles: celle de l'intentionnalité, si on considère le phénomène du point de vue de l'auteur et, si l'on se place du côté du lecteur, celle de sa capacité à interpréter les allusions discrètement disséminées par le poète dans le texte. Renonçant à aborder la seconde qui exige sans doute le recours à une histoire sociale et à une sémiologie de la lecture, je reformulerai la première de la manière suivante : les poètes donnent-ils aux lecteurs le moyen de repérer les allusions et les lieux intertextuels semés dans leurs textes? À cette question il y a au moins deux réponses : la première, qui n'est pas sans rapport avec une position prise par G.B. Conte ${ }^{32}$, est que "c'est l'œuvre elle même qui convoque un modèle de lecteur et une posture lectoriale de sa part ", l'incitant (ou non) à rechercher ou à identifier ce à quoi le texte peut faire allusion, parmi un ensemble de possibles dépendant de sa compétence. L'identification de la dimension intertextuelle de l'œuvre est donc une réponse du lecteur à une sollicitation générale de l'œuvre programmée par l'écrivain ; et que cette réponse soit valide ou non, possible ou probable, variable dans le temps ou constante, il est clair que c'est l'œuvre qui en ouvre ou non la possibilité, sans pour autant la contraindre absolument. Mais comment le fait-elle? Je vais essayer de le montrer en prenant deux exemples dont le premier est consacré à la relation que les poètes latins entretiennent avec Homère, cible privilégiée pour les critiques de la profondeur intertextuelle des épopées latines ${ }^{33}$.

Dans l'ouvrage que j'ai déjà mentionné, A. Laird considère comme une interprétation idéologique, donc contestable, l'identification par les lecteurs antiques et modernes d'un intertexte homérique chez Virgile, en arguant que la mention explicite d'Homère, de l'Iliade et de l'Odyssée, dans l'Énéide, qui aurait pu seule la rendre décisive et objective, est absente. Sa position sur le fond me paraît excessive et je ne suis pas sûr que l'exemple qu'il prend soit le meilleur. Il est vrai que le nom d'Homère et le titre de ses œuvres n'apparaissent pas dans l'Énéide - l'auraient-ils pu? -, mais ils s'y inscrivent en filigrane en de nombreux passages et notamment selon moi lors de la catabase d'Énée. Mon premier argument repose sur une proposition que j'ai déjà avancée antérieurement et consiste à voir dans l'épisode de la catabase la récriture du songe qui, d'après Lucrèce, ouvrait (?) les Annales d'Ennius : "Le récit d'Énée oppose une représentation métaphorique interne, intégrée à l'action et thématisée, de l'origine homérique de l'épopée virgilienne à la représentation externe, et naïve, d'Ennius qui a recours à la vieille fiction hésiodique, modernisée par Callimaque, du songe pour se présenter comme un alter Homerus, issu lui-même par palingénésie du paon dans lequel l'âme d'Homère avait migré ${ }^{34} »$. D'un certain point de vue, donc, le récit de la catabase offrait à Virgile l'occasion de citer Homère. Il ne le fait pas pourtant, contrairement, et c'est mon deuxième argument, à Silius qui, dans ses Punica, permet à son héros, Scipion, d'apercevoir l'aède. Alors qu'Enée, quand il pénètre avec la Sibylle dans les champs Élysées, rencontre deux figures mythiques de la poésie, Orphée et Musée, c'est auprès 
d'Homère, assimilé à une dieu, que cette même Sibylle conduit Scipion, au plus profond de son voyage descriptif. Tout se passe comme si Silius comblait par cette précision ce qu'il pointe comme un manque de la part de Virgile ou, si l'on préfère, actualisait ce qu'il considère comme virtuel et implicite chez son prédécesseur.

Du reste, le même cas se rencontre une seconde fois : il concerne Ennius, que Virgile ne mentionne pas, mais que ce même Silius évoque longuement au livre 12 des Punica :

Mais vous, Calliope, accordez à notre ouvrage de livrer à la longue suite des âges les exploits trop peu connus d'un noble guerrier, et de consacrer au poète inspiré (uati) l'honneur qui lui revient. Ennius, de l'antique race du roi Messapus, se battait au premier rang de la mêlée, et l'orgueilleux honneur du cep latin décorait sa main droite ; une rugueuse contrée, la Calabre, l'envoyait ; Rudies la vieille lui donna le jour ; maintenant Rudies n'est qu'un nom que seul son enfant fait connaître. (12, 387-397)

Ennius, que Silius désigne comme guerrier et uates, auteur comme lui d'une épopée historique, représente donc pour le poète à la fois le sujet d'une geste et un modèle voire une source poétiques. Mais chez Virgile, aussi, la relation à Ennius comme modèle et source existe, bien qu'elle se fasse de façon plus discrète: elle me paraît transparaitre ou être suggérée dans la description, empruntée à Homère, du bouclier d'Énée dont le sujet est, comme celui des Annales, « l'histoire de l'Italie et les triomphes des Romains » $(8,626)$, cette histoire romaine à venir, comme l'épopée d'Ennius ellemême ; le bouclier, non enarrabile textum $(8,625)$, m'apparaît ainsi comme l'image même de l'histoire de Rome, centrée autour des Enéades, et sa description comme le simulacre d'une épopée qui, telles les Annales, célèbre cette histoire ${ }^{35}$.

Mais est-il vrai enfin que l'Enéide ne contient aucune mention décisive et objective d'Homère, malgré le nombre élevé d'allusions possibles à l'aède grec, telle, sans doute, la description de la frise du temple de Junon qui, notons-le, fonctionne dans la fiction comme le simulacre d'un document détenant un contenu informatif : cette frise célèbre une geste héroïque dont le sujet assurément déborde celui de l'Iliade et peut-être même évoque, si l'on en croit un critique averti, la poésie cyclique postérieure à Homère (orbem, 1, 457). La lecture qu'en fait Énée, qui a ici comme ailleurs le statut de mandataire auctorial, peut être interprétée comme celle que Virgile a faite des textes dont il s'est inspiré pour composer son épopée. Mais il faut avouer que rien ne désigne de manière absolue l'intertexte homérique, bien qu'il puisse être considéré comme le plus probable.

Si l'on peut trouver cet exemple discutable, tel n'est pas le cas, selon moi, de l'épisode de la rencontre d'Énée et de la Sibylle et, plus précisément de la prophétie que lui délivre cette prêtresse en qui je suis tenté de reconnaître, malgré l'écart des noms ${ }^{36}$, cette Hérophile ou Démophile, fille d'une nymphe de l'Ida, première sibylle du dieu Apollon à Marpesos ${ }^{37}$, près de Troie. Voici ce que j'ai écrit à ce propos dans un article à paraître ${ }^{38}$ :

"S'il est vrai que cette prophétie présente des traits communs avec de véritables responsa (son style et son caractère énigmatique ${ }^{39}$ notamment), il n'est pas moins évident que son contenu informationnel et proleptique est quasiment nul : elle ne dit rien (à Enée) qui puisse le rassurer ou l'éclairer sur son avenir (c'est Anchise qui le fera ${ }^{40}$ ). Elle semble donc constituer moins un discours de la Sibylle à Enée qu'une confidence que Virgile adresse à son lecteur par son entremise, confidence qui concerne l'organisation narrative de son épopée et son statut générique. En annonçant que les guerres (horrida bella) vont succéder aux périls de la mer endurés par le héros 
(magnis pelagi periclis), elle ne fait rien d'autre que pointer la particularité essentielle du récit qui rapporte sa geste, c'est-à-dire sa structure bipartite, composée d'une odyssée et d'une iliade, structure, précisément, que les Anciens ont le plus souvent relevée pour la comparer au diptyque homérique ${ }^{41}$. La suite de la prédiction est du même ordre, puisqu'elle consiste justement à présenter la geste future d'Énée comme une iliade latine : la guerre qu'elle prédit n'est que la réplique de celle qu'Homère a chantée avec son décor (le Simois et le Xanthe, v. 88), son protagoniste (Achille, v. 89) et la cause même de la guerre, "l'hymen cherché au dehors" (v. 94), la seule différence, impliquée par le fait de la réécriture, étant le paradoxe de l'alliance des Troyens avec des Grecs (v. 97). Une telle allusion au caractère iliadique des livres 7 à 12 n'est d'ailleurs pas isolée ${ }^{42}$ : elle sera reprise, dans un cadre d'énoncé auctorial, au début du livre 7 quand Virgile, annonçant dans des termes voisins le sujet de cette partie de son épopée (horrida bella en même position finale aux vers 6,86 et 7,41$)$, précise au lecteur : maius opus moueo ( 7 , 44), laissant entendre qu'il sait, comme tous ses lecteurs, que, dans la hiérarchie des épopées modèles, l'épopée homérique de la guerre, l'Iliade, qu'il va désormais imiter, est tenue pour supérieure à l'épopée du voyage, l'Odyssée, dont il vient d'achever la reprise ".

La Sibylle est donc un personnage qui joue un double rôle, celui de prophétesse des destins du héros au niveau de l'action racontée et celui d'un modèle poétique qui illustre le rapport de l'auteur à la tradition homérique. Virgile se sert d'elle pour informer son lecteur sur l'organisation narrative de son œuvre, sur ses modèles et ses sources et, partant, sur la conception générique qu'elle illustre et les contenus axiologiques qu'elle véhicule. Si l'on admet l'hypothèse, avancée plus haut, que c'est le même personnage, vivant chez Virgile et Ovide, qui réapparaît chez Silius comme figure du monde des morts, on admettra qu'à travers elle, c'est une tradition poétique particulière, celle d'un certain type d'épopée romaine, dans la continuité d'Homère, qui se pense et se représente comme lieu d'exercice de l'intertextualité (réécriture), comme symbole de l'architextualité (statut générique) et comme figure métatextuelle sur l'art poétique (inspiration et art).

\section{Conclusion}

Par ces quelques réflexions, je me suis attaché à montrer que le souci de vraisemblance pragmatique n'est pas absent de l'écriture épique et que ses manifestations remplissent une fonction particulière au niveau des procédés de l'autoréférence, celle de convoquer, filtrer et hiérarchiser les données de la tradition considérées par le poète comme pertinentes, en ce sens que c'est par elles qu'il fonde et légitime son entreprise poétique. Ainsi défini, le protocole pragmatique pourra être considéré comme l'une des clés de la poétique auctoriale représentée par le poète dans son œuvre. 


\section{NOTES}

1. M. Robert, Livre de lectures, Paris, Grasset, 1983, pp. 83-84.

2. C. Cavillac, «Vraisemblance pragmatique et autorité fictionnelle », Poétique 101, 1995, Seuil, pp. 23-46.

3. Elle l'appelle « pacte d'illusion consentie ».

4. Voir, par exemple, le topos du manuscrit trouvé.

5. Dans l'exposé présenté lors de la journée consacrée à la vraisemblance, Séminaire « Frontières de la fiction », Lille, Institut Érasme, juin 2002.

6. Et donc sans se référer à une source réelle ou prétendue.

7. Quintilien, I.O. $(4,2,89)$ : Sed utrumcumque erit, prima sit curarum ut id quod fingemus fieri possit, deinde ut et personae et loco et tempori congruat et credibilem rationem et ordinem habeat... Il ajoute que, « si on le peut, on ajustera ce qui n'est qu'imaginaire avec quelque circonstance vraie... ».

8. Cité par C. Cavillac, art. cit., p. 27.

9. Sur ce point voir S. Rabau, "La première phrase du premier roman ", Poétique, 82, 1990, p. 131-144, à qui est empruntée l'analyse qui suit.

10. Arrien au $\mathrm{II}^{\mathrm{e}}$ siècle et Hérodien au $\mathrm{III}^{\mathrm{e}}$ feront de même.

11. Cf. aussi Polybe, è tôn progegenèmenon praxeon epistémè.

12. C. Cavillac, art. cit., p. 30.

13. Du reste, en deux occasions (au moins), le roman contient une évaluation de l'artifice autobiographique sur lequel repose sa vraisemblance pragmatique: elle se trouve dans deux récits internes (qui sont comme des mises en abyme du récit principal) eux-mêmes présentés comme des histoires vécues par le narrateur : d'abord avec l'histoire de Socrate, une histoire de zombie, racontée par un narrateur interne à deux compagnons de voyage dont l'un est Lucius (alors que ce dernier ajoute foi au récit qui lui est proposé et croit possibles les événements qu'il contient, l'autre n'y voit que mensonge et fable); et avec le récit de sorcières que fait au cours d'un banquet un milésien (!) nommé Thélyphron : une nouvelle fois Lucius se montre crédule, alors que tous les autres convives éclatent de rire (selon moi, parce qu'ils se moquent de sa crédulité) ; un peu comme devaient rire les lecteurs des Métamorphoses. Le procédé de l'artifice autobiographique y est en quelque sorte dénudé et est donné comme tel, c'est-à-dire comme faisant partie du code de la milésienne.

14. Voir aussi Properce 2, 1, 1-4.

15. L'expression est de J. Rousset, Forme et signification, Paris, 1962, p. 75 (citée par C. Cavillac).

16. Voir aussi A. Laird, Power of Expression, Expression of Power, Oxford UP, 199898, pp. 9-12.

17. « Musa Pedestre », dans Lo Spazio Letterario di Roma Antica, I, Rome, 1989, p. 311-341.

18. Dans l'exposé présenté lors de la journée consacrée à la vraisemblance, Séminaire « Frontières de la fiction », Lille, Institut Érasme, juin 2002.

19. D'où aussi sa préférence aussi pour ce qui est impossible mais vraisemblable à ce qui est possible mais non crédible (24 60a).

20. Aristote, Poétique, 9,51 b 30.

21. Cf. J. Cousin, Études sur Quintilien, I, Paris, 1935, p. 113-118. Sur Asclépiade de Myrlaea et la tripartition, cf. G. Rispoli, Lo spazio del verisimile. Il racconto, la storia e il mito, Napoli, 1988.

22. «Cette traduction du grec eikos par le latin veri similis, qui donne verosimil en espagnol (et veri similitudo, verisimilitud, employé concurremment avec semejança a verdad par le Pinciano) est un fait décisif dans l'histoire du concept » (art. cit., p. 35).

23. Et quia narrationum, excepta qua in causis utimur, tris accepimus species, fabulam, quae uersatur in tragoediis atque carminibus, non a ueritate modo, sed etiam a forma ueritatis remota, argumentum, quod 
falsum, sed uero simile comoediae fingunt, historiam, in qua est gestae rei expositio, grammaticis autem poeticas dedimus : apud rhetorem initium historica, tanto robustior quanto uerior (I.O., II, 4, 2).

24. G. Genette, Récit et Diction, Paris, Seuil, 1991, p. 66.

25. Pour l'une qui se définit comme un art de la fiction poétique, une narration poétique est une composition (poiein) représentant (mimèsis) une suite d'actions pouvant avoir lieu dans l'ordre du vraisemblable et du nécessaire, et se distingue de la chronique historique qui raconte ce qui a eu lieu réellement, même si les faits rapportés paraissent non crédibles. Pour la rhétorique, qui se définit comme un art du discours persuasif, la narration est, selon Quintilien lui-même, « l'exposé d'une chose vraie, ou donnée pour telle (expositio rei factae aut ut factae), fait dans le but de persuader...".

26. Aristote, Poétique, 9, 51 b 30.

27. Quintilien, I.O. (4, 2, 89).

28. "Narratio est rerum gestarum aut ut gestarum expositio. Narrationum genera tria sunt : unum genus est, in quo ipsa causa et omnis ratio controversiae continetur; alterum, in quo digressio aliqua extra causam aut criminationis aut similitudinis aut delectationis non alienae ab eo negotio, quo de agitur, aut amplificationis causa interponitur. Tertium genus est remotum a civilibus causis, quod delectationis causa non inutili cum exercitatione dicitur et scribitur. Eius partes sunt duae, quarum altera in negotiis, altera in personis maxime versatur. Ea, quae in negotiorum expositione posita est, tres habet partes : fabulam, historiam, argumentum. Fabula est, in qua nec verae nec veri similes res continentur, cuiusmodi est : " Angues ingentes alites, iuncti iugo..." HISTORIA est gesta res, ab aetatis nostrae memoria remota; quod genus: "Appius indixit Carthaginiensibus bellum». ARGUMENTUM est ficta res, quae tamen fieri potuit. Huiusmodi apud Terentium: "Nam is postquam excessit ex ephebis, [Sosia]...» (= fragment 238 ed. Loeb).

29. Sur cette partie, voir. A. Laird, op. cit., Oxford UP, 1998, op. cit.

30. Voir C. Calame, Le récit en Grèce ancienne, Méridiens Klincksiek, 1986, passim.

31. La tradition fonctionne donc comme le lieu d'une doxa, fondée sur le consensus, doxa dont chaque poète est à sa manière le témoin et le garant; et la vraisemblance pragmatique de son récit, l'écrivain la tire de son inscription, comme le dépositaire et l'héritier d'une lignée, dans le réseau des dires antérieurs, issus d'un dire premier, celui du fondateur grec (et de son émule latin) qui lui donne son autorité et sa légitimité. Elle ne repose plus sur la prétention à révéler un dire inouï, mais à répéter, en les intégrant, tous les dires antérieurs.

32. GB. Conte, Genres and Readers (trad. G. Most), Baltimore, 1994, p. XX.

33. Knauer G.N., Die Aeneis und Homer, in Hypomnemata 7, Göttingen, 1964.

34. D'après Ann. I, XI, Vahl3 ; cf. Lucrèce, De natura rerum, 1, 120 sq..

35. Le fait que le bouclier évoque des événements qui, vu leur date, ne pouvaient faire l'objet du récit ennien, importe peu.

36. Elle s'appelle Deiphobé chez Virgile, mais la tradition est confuse.

37. Pausanias, $10,2$.

38. « La Sibylle dans la tradition épique à Rome : Virgile, Ovide et Silius Italicus ", Actes du colloque de Rennes sur la Sibylle, novembre 2002, à paraître.

39. Elle est commentée par Virgile lui-même en ces mots : Talibus ex adyto dictis Cumaea Sibylla/ horrendas canit ambages antroque remugit/obscuris uera inuoluens... $(6,98-100)$.

40. En 6, 6, 890-92.

41. Voir. notamment Servius et Macrobe (Saturn. V, 5). Lire A. Deremetz «Énée aède. Tradition auctoriale et (re)fondation d'un genre", dans L'Histoire littéraire immanente, Entretiens de la Fondation Hardt, XLVII, Vandoeuvres-Genève, 2001, p. 144-181.

42. Voir aussi le même rapprochement en VII, 321-2 : quin idem Veneri partus suos et Paris alter/ funestaeque iterum recidiua in Pergama taedae. 


\section{AUTEUR}

ALAIN DEREMETZ

Université Charles-de-Gaulle - Lille 3 